\title{
The effect of perinatal anxiety on bronchiolitis is influenced by polymorphisms in ROS-related genes
}

\author{
Eun Lee ${ }^{1 \dagger}$, Hyoung Yoon Chang ${ }^{2 \dagger}$, Kyung-Sook Lee ${ }^{3}$, Dong In Suh ${ }^{4}$, Ho-Sung Yu ${ }^{5}$, Mi-Jin Kang ${ }^{5}$, In Ae Choi ${ }^{6}$, \\ Jinah Park ${ }^{3}$, Kyung Won Kim ${ }^{7}$, Youn Ho Shin ${ }^{8}$, Kang Mo Ahn ${ }^{9}$, Ja-Young Kwon ${ }^{10}$, Suk-Joo Choi ${ }^{11}$, Kyung-Ju Lee ${ }^{5}$, \\ Hye-Sung Won ${ }^{12}$, Song I Yang ${ }^{1}$, Young-Ho Jung ${ }^{1}$, Hyung Young Kim ${ }^{13}$, Ju-Hee Seo ${ }^{14}$, Ji-Won Kwon ${ }^{15}$, \\ Byoung-Ju Kim ${ }^{16}$, Hyo-Bin Kim ${ }^{17}$, So-Yeon Lee ${ }^{18}$, Eun-Jin Kim ${ }^{19}$, Joo-Shil Lee ${ }^{19}$, Katherine M Keyes ${ }^{20}$, Yee-Jin Shin ${ }^{21^{*}}$, \\ Soo-Jong Hong ${ }^{1 *}$ and the COCOA study group
}

\begin{abstract}
Background: Exposure to perinatal anxiety affects disease susceptibility in offspring but studies on the association between perinatal anxiety and gene polymorphisms are lacking. This study aimed to elucidate the interaction between perinatal anxiety and polymorphisms in antioxidant defense and innate immunity genes on the development of respiratory tract infections (RTIs) during early infancy.

Methods: Trait anxiety levels in 440 women were assessed by the State-Trait Anxiety Inventory during late gestation. The occurrence of RTIs, including bronchiolitis, during the first year of life was assessed by parent-reported doctor diagnosis. Polymorphisms in glutathione S-transferase P-1 (GSTP1, rs1695) and CD14 (rs2569190) were genotyped using the TaqMan assay. Copy number variations of GSTT1 were measured by real-time polymerase chain reaction.

Results: Exposure to high levels of perinatal anxiety increased the risk of bronchiolitis in the first year of life (adjusted odds ratio [aOR], 1.30; 95\% confidence interval [Cl]: 1.00-1.80), in particular among children with the AG + GG genotype of GSTP1 or the GSTT1 null genotype (aOR 3.36 and 2.79). In infants with the TC + CC genotype of CD14, high levels of perinatal anxiety were associated with an increased risk of upper RTI, lower RTI, and bronchiolitis (aOR 2.51, 4.60, and 4.31, respectively).

Conclusions: Perinatal maternal anxiety levels affect the occurrence of bronchiolitis in offspring. The effect of perinatal anxiety on the occurrence of bronchiolitis during infancy was influenced by genetic polymorphisms in antioxidant defense and innate immunity genes.
\end{abstract}

Keywords: Anxiety, Bronchiolitis, CD14, Perinatal, Glutathione S-transferase, Polymorphism, Respiratory tract infection

\section{Background}

Environmental factors during early life influence the development of the immune system and physiology, and change susceptibility to disease in later life [1]. Recent studies reveal that perinatal maternal stress and anxiety affect susceptibility to infectious diseases in later life [2]. Respiratory tract infections (RTIs) account for the

\footnotetext{
* Correspondence: yjshin@yuhs.ac; sjhong@amc.seoul.kr

${ }^{\dagger}$ Equal contributors

${ }^{21}$ Department of Psychiatry, Gangnam Severance Hospital, Yonsei University College of Medicine, 250 Seongsandong, Seodaemun-gu, Seoul 120-752, Korea 1 Department of Pediatrics, Childhood Asthma Atopy Center, Research Center for Standardization of Allergic Diseases, Asan Medical Center, University of Ulsan College of Medicine, 88 Olympic-ro 43-gil, Songpa-gu, Seoul 138-736, Korea Full list of author information is available at the end of the article
}

majority of morbidity and mortality during infancy and thus pose a worldwide burden that can be prevented by the modification of environmental factors $[3,4]$.

The mechanisms underlying the associations between exposure to perinatal maternal anxiety and an increased risk of RTIs are not fully elucidated. Perinatal maternal anxiety increases glucocorticoids in pregnant women, which can cross the placental barrier. This may affect fetal programming through immunomodulation [5], and perinatal anxiety may cause epigenetic changes in the offspring leading to an increased vulnerability to neurodevelopmental diseases in later life [6]. However, no study has directly demonstrated the possible mechanisms underlying the association between exposure to 
perinatal maternal anxiety and development of RTIs in offspring.

Perinatal anxiety and RTIs share a common pathway of oxidative stress $[7,8]$. Exposure to perinatal anxiety increases the serum levels of glucocorticoids in offspring [9], generating higher levels of reactive oxygen species (ROS). During RTIs, high levels of ROS are generated in the epithelium of the respiratory tract. This increased oxidative stress is associated with alterations in the immune response and affects fetal programming $[10,11]$.

The glutathione S-transferase (GST) subfamily plays an important role in the protection against oxidative stress by catalyzing the conjugation of many compounds with reduced glutathione [12]. Polymorphisms in the GST genes, GSTP1 (rs1695) and GSTT1, affect the ability to deal with excessive oxidative stress due to the resultant altered activity of the GST enzymes [12]. In subjects with the AG + GG genotype of GSTP1 (rs1695), the enzymatic activity of GSTP1 is partially reduced, compared to subjects with the AA genotype [12]. The null genotype of GSTT1 is associated with an absence of enzyme activity [12]. CD14 is essential for host defense because it acts as a receptor for microbial ligands during the innate immune response [13]. A functional polymorphism of CD14 (rs2569190) is associated with an enhanced immune response, and thereby affects the risk of RTIs $[14,15]$. This suggests that these genetic variants may affect the occurrence of RTIs and environmental factors may exaggerate the effects of these genetic variations.

Therefore, we hypothesized that there are associations between exposure to perinatal anxiety and genetic variants involved in antioxidant defense and innate immunity, and these interactions influence the RTI risk in offspring, especially during early life. The first aim of this study was to examine the association between perinatal anxiety and RTI occurrence during the first year of life. Second, the effect of the interaction between exposure to perinatal anxiety and genetic variants of some GST subfamily genes and CD14 (rs2569190) on the occurrence of RTIs was explored.

\section{Methods}

\section{Participants}

Data was used from the COhort for Childhood Origin of Asthma and allergic disease (COCOA), a prospective birth cohort study that aimed to investigate the effects of environmental and genetic factors during early life on the development of allergic diseases and children's health [16-18]. Women in the third trimester of pregnancy were enrolled from January 2009 to September 2011. The questionnaire used in this study included items on the State-Trait Anxiety Inventory (STAI). Pregnant women with pregnancy-associated complications (gestational diabetes and pregnancy-induced hypertension), high risk for early delivery, and delivery earlier than 36 weeks have been excluded. Neonates were excluded if they needed oxygen therapy, or had congenital anomalies including congenital diaphragmatic hernia, congenital heart disease, congenital lung diseases, or severe systemic diseases. More details on this cohort study are described elsewhere [19].

Among 734 eligible women, 71 refused to participate, 4 were lost to follow up, and 28 were excluded due to preterm delivery. Among a total of 631 (86\%), 440 (69.7\%) completed the STAI questionnaire. Study participants did not differ from those women not included in the study with regard to key demographic covariates, except for the season of offspring's birth (Table 1). Information on potential confounders was obtained from a parentalreported questionnaire at the 36th week of pregnancy and medical records at the child's birth. Questionnaires assessed maternal health problems including allergic diseases (atopic dermatitis, allergic rhinitis, or asthma), socioeconomic status (income and educational levels), and prenatal environmental factors such as tobacco smoking exposure at 36 weeks of gestation. The newborn's sex and weight, gestational age, season of birth, and health conditions were obtained by a questionnaire after birth.

Written informed consent was obtained from all women and the study was approved by Asan Medical Center (IRB No. 2008-0616), Samsung Medical Center (IRB No. 2009-02-021), Yonsei University (IRB No. 4-2008-0588), and CHA Medical Center (IRB No. 2010-010).

\section{Anxiety assessment}

Data on perinatal maternal anxiety were obtained by self-report at the 36th week of pregnancy. Instead of using STAI, the Korean version of STAI (K-STAI) was used, which consists of two subscales, namely State Anxiety (anxiety about an event) and Trait Anxiety (anxiety levels as a personal characteristic). The Trait Anxiety subscale (STAI-T) was used in this study because it reflects the baseline anxiety levels of the subjects rather than a transient anxiety state. STAI-T is a 20 item questionnaire scored on a four-point Likert-type scale that measures a general tendency to be anxious [20]. Scores range from 20 to 80 , with a higher score indicating a more severe anxiety level. K-STAI exhibits excellent psychometric properties [21] and its internal consistency has been reported as having a Cronbach's $\alpha$ coefficient of 0.91 [22]. In this study, the reliability coefficient (Cronbach's $\alpha$ ) of STAI-T was 0.92 .

\section{Assessment of 1 year outcome variables}

The infants were examined at 1 year of age with at least one parent present at follow-up visits. RTIs were assessed by parent-reported doctor diagnosis during the first year of life. RTIs were further categorized as upper (URTIs) and lower (LRTIs) RTIs. URTIs included common colds, 
Table 1 Characteristics of the study population and subjects not included in the current analysis

\begin{tabular}{|c|c|c|c|c|c|}
\hline & \multicolumn{2}{|c|}{ Women included in this study } & \multicolumn{2}{|c|}{ Women not included in this study } & \multirow[t]{2}{*}{ P-value } \\
\hline & $n$ & Mean \pm SD or $n(\%)$ & $n$ & Mean \pm SD or $n(\%)$ & \\
\hline Covariates (mother) & 440 & & 193 & & \\
\hline Maternal age (years) & & $32.5 \pm 3.55$ & & $32.4 \pm 3.45$ & 0.736 \\
\hline $\mathrm{BMI}\left(\mathrm{kg} / \mathrm{m}^{2}\right)$ & & $20.7 \pm 2.67$ & & $20.7 \pm 2.60$ & 0.754 \\
\hline \multicolumn{6}{|l|}{ Maternal educational level } \\
\hline Secondary school & & $31(7.0 \%)$ & & $21(10.9 \%)$ & 0.202 \\
\hline College or University & & $289(65.7 \%)$ & & $116(60.1 \%)$ & \\
\hline Graduate school & & $120(27.3 \%)$ & & $56(29.0 \%)$ & \\
\hline Environmental tobacco exposure & & $265(60.8 \%)$ & & $105(54.4 \%)$ & 0.171 \\
\hline History of any allergic disorder (AD, AR, Asthma) & & $124(28.2 \%)$ & & $59(30.7 \%)$ & 0.568 \\
\hline Covariates (father) & 410 & & & & \\
\hline History of any allergic disorder (AD, AR, Asthma) & & $115(26.1 \%)$ & & $51(26.4 \%)$ & 0.939 \\
\hline Covariates (infant) & 440 & & 193 & & \\
\hline Sex: Girl & & $217(49.3 \%)$ & & $85(44.2 \%)$ & 0.227 \\
\hline Birth weight (g) & & $3143.9 \pm 422.01$ & & $3198.3 \pm 395.43$ & 0.153 \\
\hline \multicolumn{6}{|l|}{ Season of birth } \\
\hline Spring & & $117(26.6 \%)$ & & $28(14.5 \%)$ & $<0.001$ \\
\hline Summer & & $117(26.6 \%)$ & & $26(13.5 \%)$ & \\
\hline Autumn & & $96(21.8 \%)$ & & $91(47.2 \%)$ & \\
\hline Winter & & $110(25.0 \%)$ & & $48(24.9 \%)$ & \\
\hline Mode of delivery: Caesarean section & & $134(34.4 \%)$ & & $68(35.2 \%)$ & 0.793 \\
\hline Gestational age (months) & & $39.2 \pm 1.08$ & & $39.2 \pm 1.09$ & 0.968 \\
\hline Presence of siblings & & $198(45.0 \%)$ & & $83(43.0 \%)$ & 0.705 \\
\hline Daycare attendance during the first year of life & & $105(23.9 \%)$ & & $43(22.3 \%)$ & 0.740 \\
\hline Exposure to perinatal maternal anxiety & 440 & & N/A & & \\
\hline STAl score & & $41.0 \pm 8.47(20-71)$ & & & \\
\hline \multicolumn{6}{|c|}{ Physician diagnosed respiratory tract infection during the first year of life } \\
\hline Upper respiratory tract infection & & $337(76.6 \%)$ & & & \\
\hline Lower respiratory tract infection & & $65(14.8 \%)$ & & & \\
\hline Bronchiolitis & & $48(10.9 \%)$ & & & \\
\hline
\end{tabular}

$A D$, atopic dermatitis; $A R$, allergic rhinitis; $N / A$, not applicable; STAl, State-Trait Anxiety Inventory.

sinusitis, otitis media, and croup; LRTIs included pneumonia, tracheobronchitis, and bronchiolitis.

\section{DNA isolation and genotyping}

Genomic DNA was extracted from the buffy coat of the cord blood at delivery using the Gentra ${ }^{\circ}$ Puregene $^{\circ}$ Blood kit (Qiagen, Maryland, USA), as recommended by the manufacturer. Genotyping of CD14 (rs2569190) and GSTP1 (rs1695) was conducted using a TaqMan assay (ABI, Foster City, CA, USA). Assay identification numbers for CD14 and GSTP1 were C_16043997_10 and C_3237198_20, respectively. Copy number variations (CNV) of GSTM1 and GSTT1 were measured by realtime polymerase chain reaction (RT-PCR). The primers and probe for GSTM1 and GSTT1 were synthesized according to Brasch-Andersen $\mathrm{C}$ et al. [23]. Reactions were performed as a triplex, with RNAse $\mathrm{P}$ as the reference gene. The allele frequencies of the polymorphisms in GSTP1 (rs1695), GSTM1, GSTT1, and CD14 (rs2569190) were in Hardy-Weinberg equilibrium $(P>0.1)$.

\section{Statistical analysis}

The association between perinatal maternal anxiety levels and RTIs (URTIs, LRTIs, and bronchiolitis) during the first year of life was examined using logistic regression. Adjustments were made for baseline demographic factors (child's sex and maternal age) as well as known risk factors for respiratory infections, including maternal educational level, exposure to tobacco smoking during the first year, and presence of maternal and paternal allergic diseases [24,25]. 
The standard deviation (SD) for the STAI score in the regression analyses was 8.47. Parameter estimates were multiplied by the SD of the STAI score, and for $95 \%$ confidence interval (CI), the following equation was used: BETA*(SD of STAI score) $\pm 1.96 * 6^{*}$ (SD of STAI score). In logistic regression analysis, the estimates were exponentiated to obtain the estimate and 95\% CI of the odds ratio (OR).

The combined effects of perinatal anxiety and genetic polymorphisms on RTI risk were investigated by multivariate logistic regression analysis. To analyze the combined effect of the two factors, STAI scores were divided into two categories on the basis of mean levels and genetic polymorphisms were classified according to GSTT1 null or present, GSTM1 null or present, and polymorphisms of GSTP1 (rs1695) and CD14 (rs2569190). All statistical analyses were performed using SAS for Windows (version 9.2).

\section{Results}

\section{Demographics}

Table 1 summarizes the descriptive statistics across the total sample. There were no significant differences in maternal age, maternal education level, infants' sex, mode of delivery, presence of infants' siblings, and daycare attendance of infants between pregnant women included in this study and those excluded. Overall, the mean maternal age was 32.5 years. Exposure to tobacco smoking during pregnancy was reported by $60.8 \%$ of the women. In mothers, $28.2 \%$ had a history of allergic disease, whereas in spouses the proportion was $26.1 \%$. The STAI score demonstrated a normal distribution with mean \pm SD of $41.0 \pm 8.47$. Approximately half $(49.3 \%)$ of the offspring were girls, with a mean gestational age of 39.2 weeks. Births were evenly distributed between the four seasons (26.6\% in spring, $26.6 \%$ in summer, $21.8 \%$ in autumn, and $25.0 \%$ in winter). Approximately one third of births (34.4\%) were delivered by caesarean section.

\section{Association between perinatal maternal anxiety and development of RTIs during the first year of life}

High levels of perinatal maternal anxiety were associated with an increased risk of LRTIs, especially bronchiolitis, but not with total URTIs (Table 2). For a 1 SD increase in STAI score, the odds of LRTI risk during the first year of life increased by 1.35 times (95\% CI: $1.01-1.78)$. The crude association between perinatal anxiety levels and the development of bronchiolitis was weakly significant (OR 1.35, 95\% CI: 1.00-1.81; adjusted OR [aOR] 1.30, 95\% CI: 1.00-1.80). However, perinatal anxiety did not increase the risk of URTIs (aOR 1.10, 95\% CI: 0.87-1.39). In addition, high levels of perinatal maternal anxiety were not significantly associated with the occurrence of other subtypes of RTIs except bronchiolitis. Figure 1 represents
Table 2 Odds ratio and $95 \%$ confidence interval for perinatal anxiety predicting RTls at age 1 year

\begin{tabular}{lllllll}
\hline Exposure & Outcome & \multicolumn{2}{c}{ Unadjusted } & & \multicolumn{2}{c}{ Adjusted $^{*}$} \\
\cline { 3 - 4 } \cline { 7 - 7 } & & OR $^{\dagger}$ & $\mathbf{9 5 \% ~ C l}$ & & OR & $\mathbf{9 5 \% ~ C l}$ \\
\hline STAI (SD) & URTI & 1.04 & $(0.84-1.30)$ & & 1.10 & $(0.87-1.39)$ \\
& LRTI & $1.31^{\ddagger}$ & $(1.01-1.71)$ & & $1.35^{\ddagger}$ & $(1.01-1.78)$ \\
& Bronchiolitis & $1.35^{\ddagger}$ & $(1.00-1.81)$ & & $1.30^{\S}$ & $(1.00-1.80)$ \\
\hline
\end{tabular}

$C l$, confidence interval; $O R$, odds ratio; $R T I$, respiratory tract infection;

$S D$, standard deviation; STAI, State-Trait Anxiety Inventory.

*Adjusted for child's sex, season of birth, maternal age, education level,

exposure to tobacco smoking during the first year of life, history of maternal

allergic diseases and history of any paternal allergic diseases (atopic

dermatitis, allergic rhinitis, or asthma).

${ }^{\dagger}$ Logistic regression models were used to calculate OR and $95 \% \mathrm{Cl}$.

${ }^{\ddagger} p$-value $<0.05$.

${ }^{\S} p$-value $<0.10$.

the predicted probability of bronchiolitis at 1 year of age for each observed value of perinatal anxiety score in the multivariate model.

Combined effect between perinatal maternal anxiety levels and GSTP1 (rs1695), GSTT1, GSTM1 polymorphisms on the development of RTIs during the first year of life Genetic polymorphisms in GSTP1 (rs1695), GSTT1 or GSTM1 did not increase the risk of any types of RTIs (data not shown). Exposure to high levels of perinatal anxiety was associated with an increased risk for LRTIs, especially bronchiolitis, in infants with the AG+GG genotype of GSTP1 (rs1695), compared to infants with exposure to low levels of perinatal anxiety and with the AA genotype (aOR 2.61, 95\% CI: 1.09-6.22; aOR 3.36, 95\% CI: 1.25-9.03, respectively) (Table 3). Analysis of the three genotypes showed that, infants with the GG genotype of GSTP1 (rs1695) had a significantly increased risk of LRTIs, especially bronchiolitis, during the first year of life (aOR 22.04, 95\% CI: 4.79-101.47; aOR 14.61, 95\% CI: 3.23-66.11, respectively) (Additional file 1: Figure S1).

In addition, infants with both the GSTT1-null genotype and exposure to high levels of perinatal anxiety were associated with an increased risk for LRTIs, especially bronchiolitis, compared to infants with both the GSTT1-present genotype and exposure to low levels of perinatal anxiety (aOR 2.47, 95\% CI: 1.04-5.89; aOR 2.79, 95\% CI: 1.05-7.43) (Table 4). In terms of GSTM1, exposure to high levels of perinatal anxiety increased the risk of LRTIs during the first year of life, regardless of the genotypes (present genotype: aOR 2.95, 95\% CI: 1.06-8.17; null genotype: aOR 2.60, 95\% CI: 1.01-6.66) (Additional file 2: Table S1).

Combined effect between exposure to perinatal maternal anxiety and CD14 (rs2569190) polymorphism on the development of RTls during the first year of life Infants with the TT genotype of CD14 (rs2569190) had an increased risk of URTIs and LRTIs, especially bronchiolitis, 


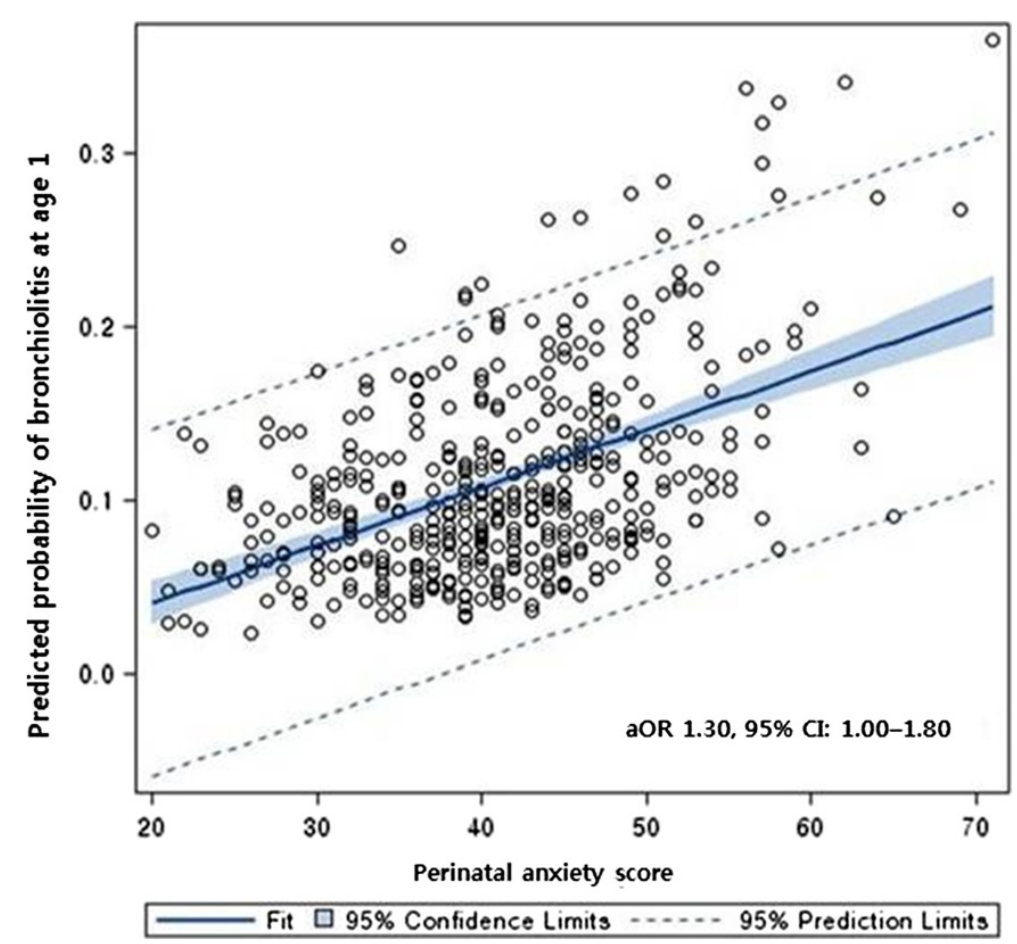

Figure 1 Relationship between perinatal maternal anxiety levels and predicted probability of bronchiolitis at 1 year of age.

if they were exposed to high levels of perinatal anxiety, compared with infants exposed to low levels of perinatal anxiety (aOR 2.51, 95\% CI: 1.01-6.24; aOR 4.60, 95\% CI: 1.29-16.41; aOR 4.31, 95\% CI: 1.17-15.79, respectively) (Additional file 3: Table S2).

\section{Discussion}

Main findings

In this study, a positive association was found between exposure to high levels of perinatal anxiety and development of RTIs in offspring during the first year of life.
The risk of LRTIs, especially bronchiolitis, during early infancy was influenced by additive effects between exposure to perinatal anxiety and polymorphisms in antioxidantrelated genes. To the best of our knowledge, this is the first study examining the gene-environment interactions with respect to perinatal anxiety on the development of RTIs during early life.

Exposure to high levels of perinatal anxiety affected the development of RTIs, especially bronchiolitis, differently during infancy, depending on the polymorphisms of ROS detoxification genes such as GSTP1 (rs1695) and GSTT1.

Table 3 Interactions between maternal perinatal anxiety (STAI score) and GSTP1 (rs1695) polymorphism on the development of respiratory tract infection during the first year of life

\begin{tabular}{|c|c|c|c|c|c|c|c|c|c|c|}
\hline \multirow[b]{2}{*}{ STAI } & \multirow[b]{2}{*}{ GSTP1 } & \multicolumn{3}{|c|}{ URTI $^{*}$} & \multicolumn{3}{|c|}{ LRTI $^{\dagger}$} & \multicolumn{3}{|c|}{ Bronchiolitis } \\
\hline & & $\begin{array}{c}\text { URTI } \\
\text { diagnosis (-) }\end{array}$ & $\begin{array}{c}\text { URTI } \\
\text { diagnosis (+) }\end{array}$ & $\begin{array}{c}\mathrm{aOR}^{\mp} \\
(95 \% \mathrm{Cl})\end{array}$ & $\begin{array}{c}\text { LRTI } \\
\text { diagnosis (-) }\end{array}$ & $\begin{array}{c}\text { LRTI } \\
\text { diagnosis (+) }\end{array}$ & $\begin{array}{c}\mathrm{aOR}^{\ddagger} \\
(95 \% \mathrm{Cl})\end{array}$ & $\begin{array}{l}\text { Bronchiolitis } \\
\text { diagnosis (-) }\end{array}$ & $\begin{array}{l}\text { Bronchiolitis } \\
\text { diagnosis (+) }\end{array}$ & $\begin{array}{c}\mathrm{aOR}^{\ddagger} \\
95 \% \mathrm{Cl}\end{array}$ \\
\hline $\operatorname{low}(\leq 41)$ & AA & 36 & 111 & 1 & 133 & 14 & 1 & 138 & 9 & 1 \\
\hline low $(\leq 41)$ & $A G+G G$ & 17 & 43 & $\begin{array}{c}1.10 \\
(0.53-2.27)\end{array}$ & 51 & 9 & $\begin{array}{c}1.66 \\
(0.64-4.26)\end{array}$ & 52 & 8 & $\begin{array}{c}2.14 \\
(0.74-6.15)\end{array}$ \\
\hline high $(>41)$ & AA & 31 & 90 & $\begin{array}{c}1.25 \\
(0.68-2.30)\end{array}$ & 101 & 20 & $\begin{array}{c}1.99 \\
(0.92-4.29)\end{array}$ & 106 & 15 & $\begin{array}{c}2.07 \\
(0.83-5.16)\end{array}$ \\
\hline high $(>41)$ & $A G+G G$ & 13 & 53 & $\begin{array}{c}1.91 \\
(0.86-4.25)\end{array}$ & 52 & 14 & $\begin{array}{c}2.61 \\
(1.09-6.22)\end{array}$ & 54 & 12 & $\begin{array}{c}3.36 \\
(1.25-9.03)\end{array}$ \\
\hline
\end{tabular}

$a O R$, adjusted odds ratio; $C l$, confidence interval; GSTP1, glutathione S-transferase P1; $L R T I$, lower respiratory tract infection; $R T I$, respiratory tract infection; URTI, upper respiratory tract infection; STAl, State-Trait Anxiety Inventory.

*URTI includes common colds, sinusitis, otitis media, and croup.

${ }^{\dagger} \mathrm{LRTI}$ includes pneumonia, tracheobronchitis, and bronchiolitis.

${ }^{\ddagger}$ Adjusted for infant's sex, season of birth, maternal age, education level, prenatal exposure to tobacco smoking, history of any maternal allergic diseases and history of any paternal allergic diseases (atopic dermatitis, allergic rhinitis, or asthma). 
Table 4 Interactions between maternal perinatal anxiety (STAI score) and copy number variation of GSTT1 on the development of respiratory tract infection during the first year of life

\begin{tabular}{|c|c|c|c|c|c|c|c|c|c|c|}
\hline \multirow[b]{2}{*}{ STAI } & \multirow[b]{2}{*}{ GSTT1 } & \multicolumn{3}{|c|}{ URTI* } & \multicolumn{3}{|c|}{$\mathrm{LRTI}^{+}$} & \multicolumn{3}{|c|}{ Bronchiolitis } \\
\hline & & $\begin{array}{c}\text { URTI diagnosis } \\
(-), \mathbf{n}\end{array}$ & $\begin{array}{l}\text { URTI diagnosis } \\
(+), \mathrm{n}\end{array}$ & $\begin{array}{c}\mathrm{aOR}^{\neq} \\
(95 \% \mathrm{Cl}) \\
\end{array}$ & $\begin{array}{l}\text { LRTI diagnosis } \\
(-), \mathrm{n}\end{array}$ & $\begin{array}{l}\text { LRTI diagnosis } \\
(+), \mathrm{n}\end{array}$ & $\begin{array}{c}\mathrm{aOR}^{\neq} \\
(95 \% \mathrm{Cl}) \\
\end{array}$ & $\begin{array}{c}\text { Bronchiolitis } \\
\text { diagnosis }(-), \mathbf{n}\end{array}$ & $\begin{array}{c}\text { Bronchiolitis } \\
\text { diagnosis }(+), \mathbf{n}\end{array}$ & $\begin{array}{c}\mathrm{aOR}^{\neq} \\
(95 \% \mathrm{Cl}) \\
\end{array}$ \\
\hline low $(\leq 41)$ & $C N V \geq 1$ & 16 & 76 & 1 & 82 & 10 & 1 & 85 & 7 & 1 \\
\hline low $(\leq 41)$ & $C N V=0$ & 37 & 76 & $0.43(0.21-0.88)$ & 102 & 11 & $0.86(0.33-2.21)$ & 104 & 9 & $0.97(0.33-2.83)$ \\
\hline high (>41) & $C N V \geq 1$ & 22 & 65 & $0.66(0.30-1.45)$ & 75 & 12 & $1.29(0.50-3.33)$ & 79 & 8 & $1.13(0.37-3.34)$ \\
\hline high (>41) & $C N V=0$ & 22 & 77 & $1.02(0.46-2.26)$ & 77 & 22 & $2.47(1.04-5.89)$ & 80 & 19 & $2.79(1.05-7.43)$ \\
\hline
\end{tabular}


The combined effect of exposure to perinatal maternal anxiety and ROS detoxification gene polymorphisms on the occurrence of LRTIs, especially bronchiolitis, may be partially attributable to the increased ROS generation caused by high levels of perinatal maternal anxiety. Perinatal anxiety increases glucocorticoid levels in both mother and fetus, and this induces superoxide production [26]. The capacity to effectively detoxify ROS is affected by polymorphisms of ROS detoxification genes $[27,28]$. The increased risk of LRTIs, especially bronchiolitis, in infants with the AG + GG genotype of GSTP1 (rs1695) or the GSTT1-null genotype after exposure to high levels of perinatal anxiety may be explained by the reduced enzymatic activity in the AG + GG genotype of GSTP1 (rs1695) and by the loss of enzymatic activity in the GSTT1-null genotype [27,28]. Since GST subfamily genes are expressed mainly in the respiratory tract and oxidative stress is increased during LRTIs, especially bronchiolitis [29], genetic variants of the GST subfamily may influence the risk of LRTIs, especially bronchiolitis.

\section{Possible underlying mechanisms}

Psychological stress and anxiety may affect the balance of oxidant and antioxidant states and thereby influence an individual's health [30,31]. In response to stress, serum glucocorticoid levels rise to modulate the stress response [32]. Glucocorticoid levels affect antioxidant enzyme levels, and antioxidant enzyme activity in the lungs begins in late gestation $[33,34]$. Considering the impact of ROS in fetal programming and variants of ROS-related genes on the capacity to detoxify ROS $[11,12]$, the increased susceptibility to RTIs in offspring exposed to perinatal anxiety might be explained by their decreased ability to mitigate the oxidative stress generated during RTIs [35].

An experimental study showed that prenatal maternal stress influences epigenetic re-programming by altering the expression of microRNAs involved in the stress response, oxidative stress, and metabolism [6]. In addition, prenatal anxiety-related immune and oxidative responses cause telomere erosion, possibly increasing the risk of infection [36,37]. On the basis of these findings, we suggest that perinatal anxiety influences the susceptibility to RTIs in offspring during critical periods in combination with genetic variants of ROS-related genes or through epigenetic mechanisms. Additional studies are needed to clarify the underlying mechanisms.

Previous studies showed that the T allele of CD14-159C/ $\mathrm{T}$ is associated with an enhanced transcriptional activity and increased soluble CD14 levels, which are markers of monocyte and macrophage activation [38,39]. The positive relationship between perinatal anxiety level and risk of RTIs in infants with the TT genotype of CD14 (rs2569190) might be due to the essential role of CD14 as an initiator of innate immunity against viral infections in the respiratory tract [14]. Alterations in cellular and humoral immune responses caused by the interactions between exposure to high levels of perinatal maternal stress and genetic variants of CD14 might partially explain these findings $[40,41]$.

In this study, it was proposed that perinatal maternal anxiety differentially affects the risk of RTIs depending on their anatomic location in the respiratory tract. Increased glucocorticoid levels during the fetal period may affect the development and function of the lungs during early life [42]. Differences in defense mechanisms, and the more severe characteristics of LRTIs than URTIs, may account for the increased risk of LRTIs $[43,44]$. In addition, functional differences attributable to the genetic variants in ROS-related genes may add an increased susceptibility to LRTIs, especially bronchiolitis, in affected infants [27,41].

Hospitalization expenditures because of bronchiolitis during infancy has increased [45]. A history of bronchiolitis in infancy is associated with adverse respiratory outcome such as reduced lung function and development of asthma in later life $[46,47]$. With the prevention of bronchiolitis during early life, health care costs and adverse respiratory health can be avoided.

\section{Potential limitations}

The current study has a few limitations. First, the sample size in this study is relatively small because this study is an ongoing prospective birth cohort study. Although the statistical power was weak to analyze the associations with respect to each type of LRTI except bronchiolitis due to the small sample size, this study indicates the importance of the effect of perinatal maternal anxiety on the occurrence of RTIs during early life. Further large-scale studies are needed to validate the association between perinatal anxiety and LRTIs during infancy, especially bronchiolitis, which is associated with adverse respiratory outcomes such as asthma in later life. Second, mechanisms underlying the additive effects between perinatal anxiety and variants of antioxidant defense genes on the occurrence of LRTIs, especially bronchiolitis, were not explored. However, previously reported findings were used to try and explain the underlying pathophysiology. Also, only one single nucleotide polymorphism (SNP) was analyzed for each gene; however, this selection was based on previous studies demonstrating an association between these SNPs and the development of respiratory illnesses [29,34,38]. Finally, approximately $30 \%$ of the participants did not report their anxiety status and were thus excluded from the analysis.

\section{Conclusion}

This study suggests that exposure to perinatal maternal anxiety is a significant risk factor for the development of bronchiolitis in the first year of life. It indicates that 
variants in antioxidant defense genes modulate the effect of in utero exposure to perinatal anxiety on infant susceptibility to bronchiolitis during critical periods. In view of the fact that bronchiolitis during early infancy is an economic and medical burden and is associated with an increased risk of recurrent wheezing and asthma in later life, efforts to decrease perinatal anxiety may help prevent bronchiolitis during infancy, especially in genetically susceptible infants.

\section{Additional files}

Additional file 1: Figure S1. Effect of GSTP1 (rs1695) polymorphisms on respiratory tract infections (RTIS) according to perinatal maternal anxiety levels. (A) The risk of upper RTIs (URTIs) during the first year of life. (B) The risk of lower RTIS (LRTIS) during the first year of life. (C) The risk of bronchiolitis during the first year of life.

Additional file 2: Table S1. Interactions between maternal perinatal stress (STAI score) and copy number variations of GSTM1 on the development of respiratory tract infection during the first year of life.

Additional file 3: Table S2. Interactions between maternal perinatal stress (STAl score) and CD14 (rs2569190) polymorphism on the development of respiratory tract infection during the first year of life.

\section{Abbreviations}

$\mathrm{Cl}$ : Confidence interval; COCOA: COhort for childhood origin of asthma and allergic disease; GSTM1: Glutathione S-transferase M1; GSTP1: Glutathione S-transferase P1; GSTT1: Glutathione S-transferase T1; LRTIs: Lower respiratory tract infections; PCR: Polymerase chain reaction; ROS: Reactive oxygen species; RSV: Respiratory syncytial virus; RTI: Respiratory tract infection; SD: Standard deviation; STAI: State-Trait Anxiety Inventory; URTIs: Upper respiratory tract infections.

\section{Competing interests}

The authors declare that they have no competing interests.

\section{Authors' contributions}

EL and HYC contributed to the analysis and interpretation of data and drafting of the manuscript. KSL, JP, and IAC contributed to the collection of data on perinatal maternal anxiety. DIS, KWK, YHS, KMA, EJK, and JSL contributed to the construction of this cohort study and conducted the follow up. JYK, S-JC, K-JL, and $\mathrm{H}-\mathrm{SW}$ contributed to the enrolment of pregnant women in the birth cohort study. M-JK and H-SY performed the genetic studies and analyzed the data. SIY, YHJ, HYK, JHS, JWK, BJK, HBK, SYL, and KMK contributed to the thorough review of the manuscript and the acquisition of data. SJ Hong and YJ Shin contributed to the conception of this study, its design, and interpretation of the data as co-corresponding authors. All authors read and approved the final manuscript.

\section{Authors' information}

Eun Lee and Hyoung Yoon Chang as co-first authors.

\section{Acknowledgements}

This research was supported by funds (2008-E33030-00, 2009-E33033-00. 2011-E33021-00, 2012-E33012-00) from the Research of Korea Centers for Disease Control and Prevention. The authors thank JY Shim, WK Kim, GC Jang, and $\mathrm{HJ}$ Yang for their valuable support during this study. In addition, the authors are grateful to the research nurses for their continuous collaboration over the years. We thank to SC Yun for statistics advice.

\section{Author details}

'Department of Pediatrics, Childhood Asthma Atopy Center, Research Center for Standardization of Allergic Diseases, Asan Medical Center, University of Ulsan College of Medicine, 88 Olympic-ro 43-gil, Songpa-gu, Seoul 138-736, Korea. ${ }^{2}$ Department of Psychiatry, Ajou University College of Medicine, Gyeonggi-do, Korea. ${ }^{3}$ Department of Rehabilitation, Hanshin University, Gyeonggi-do, Korea. ${ }^{4}$ Department of Pediatrics, Seoul National University College of Medicine, Seoul,
Korea. ${ }^{5}$ Asan Institute for Life Sciences, University of Ulsan College of Medicine, Seoul, Korea. ${ }^{6}$ Sewon Infant Child Development Center, Seoul, Korea. ${ }^{7}$ Department of Pediatrics, Severance Children's Hospital, College of Medicine, Yonsei University, Seoul, Korea. ${ }^{8}$ Department of Pediatrics, CHA Medical Center, CHA University School of Medicine, Seoul, Korea. ${ }^{9}$ Department of Pediatrics, Samsung Medical Center, Sungkyunkwan University School of Medicine, Seoul, Korea. ${ }^{10}$ Department of Obstetrics and Gynecology, Yonsei University College of Medicine, Seoul, Korea. ${ }^{11}$ Department of Obstetrics and Gynecology, Samsung Medical Center, Sungkyunkwan University School of Medicine, Seoul, Korea. ${ }^{12}$ Department of Obstetrics and Gynecology, Asan Medical Center, University of Ulsan College of Medicine, Seoul, Korea. ${ }^{13}$ Department of Pediatrics, Pusan National University Yangsan Hospital, Yangsan, Korea. ${ }^{14}$ Department of Pediatrics, Korea Cancer Center Hospital, Seoul, Korea. ${ }^{15}$ Department of Pediatrics, Seoul National University Bundang Hospital, Seongnam, Korea. ${ }^{16}$ Department of Pediatrics, Inje University Haeundae Paik Hospital, Busan, Korea. ${ }^{17}$ Department of Pediatrics, Inje University Sanggye Paik Hospital, Seoul, Korea. ${ }^{18}$ Department of Pediatrics, Hallym University Sacred Heart Hospital, Hallym University College of Medicine, Anyang, Korea. ${ }^{19}$ Allergy TF, Department of Immunology and Pathology, Korea National Institute of Health, Cheongwon, Korea. ${ }^{20}$ Department of Epidemiology, Columbia University Mailman School of Public Health, New York, NY, USA. ${ }^{21}$ Department of Psychiatry, Gangnam Severance Hospital, Yonsei University College of Medicine, 250 Seongsandong, Seodaemun-gu, Seoul 120-752, Korea.

Received: 13 May 2014 Accepted: 23 September 2014

Published: 29 September 2014

\section{References}

1. Hanson M, Gluckman P, Nutbeam D, Hearn J: Priority actions for the noncommunicable disease crisis. Lancet 2011, 378:566-567.

2. Tegethoff M, Greene N, Olsen J, Schaffner E, Meinlschmidt G: Stress during pregnancy and offspring pediatric disease: A National Cohort Study. Environ Health Perspect 2011, 119:1647-1652

3. Tregoning JS, Schwarze J: Respiratory viral infections in infants: causes, clinical symptoms, virology, and immunology. Clin Microbiol Rev 2010, 23:74-98.

4. Beijers $R$, Jansen J, Riksen-Walraven $M$, de Weerth C: Maternal prenatal anxiety and stress predict infant illnesses and health complaints. Pediatrics 2010, 126:e401-e409.

5. Merlot E, Couret D, Otten W: Prenatal stress, fetal imprinting and immunity. Brain Behav Immun 2008, 22:42-51.

6. Zucchi FC, Yao Y, Ward ID, Inytskyy Y, Olson DM, Benzies K, Kovalchuk I, Kovalchuk O, Metz GA: Maternal stress induces epigenetic signatures of psychiatric and neurological diseases in the offspring. PLoS One 2013, 8:e56967.

7. Hosakote YM, Liu T, Castro SM, Garofalo RP, Casola A: Respiratory syncytial virus induces oxidative stress by modulating antioxidant enzymes. Am J Respir Cell Mol Biol 2009, 41:348-357.

8. Charil A, Laplante DP, Vaillancourt C, King S: Prenatal stress and brain development. Brain Res Rev 2010, 65:56-79.

9. Erni K, Shaqiri-Emini L, La Marca R, Zimmermann R, Ehlert U: Psychobiological effects of prenatal glucocorticoid exposure in 10-year-old-children. Front Psychiatr 2012, 3:104.

10. Diz-Chaves Y, Pernia O, Carrero P, Garcia-Segura LM: Prenatal stress causes alterations in the morphology of microglia and the inflammatory response of the hippocampus of adult female mice. J Neuroinflammation 2012, 9:71.

11. Thompson LP, Al-Hasan Y: Impact of oxidative stress in fetal programming. J Pregnancy 2012, 2012:582748.

12. Hayes JD, Strange RC: Glutathione S-transferase polymorphisms and their biological consequences. Pharmacology 2000, 61:154-166.

13. Kurt-Jones EA, Popova L, Kwinn L, Haynes LM, Jones LP, Tripp RA, Walsh EE, Freeman MW, Golenbock DT, Anderson L, Finberg RW: Pattern recognition receptors TLR4 and CD14 mediate response to respiratory syncytial virus. Nat Immunol 2000, 1:398-401.

14. Inoue $Y$, Shimojo N, Suzuki Y, Campos Alberto EJ, Yamaide A, Suzuki S, Arima T, Matsuura T, Tomiita M, Aoyagi M, Hoshioka A, Honda A, Hata A Kohno Y: CD14-550 C/T, which is related to the serum level of soluble CD14, is associated with the development of respiratory syncytial virus bronchiolitis in the Japanese population. J Infect Dis 2007, 195:1618-1624.

15. Wiertsema SP, Khoo SK, Baynam G, Veenhoven RH, Laing IA, Zielhuis GA, Rijkers GT, Goldblatt J, Lesouef PN, Sanders EA: Association of CD14 
promoter polymorphism with otitis media and pneumococcal vaccine responses. Clin Vaccine Immunol 2006, 13:892-897.

16. Kim HB, Ahn KM, Kim KW, Shin YH, Yu J, Seo JH, Kim HY, Kwon JW, Kim BJ, Kwon JY, Kim BJ, Choi SJ, Lee KJ, Park HJ, Won HS, Hong SJ: Cord blood cellular proliferative response as a predictive factor for atopic dermatitis at 12 months. J Korean Med Sci 2012, 27:1320-1326.

17. Shin YH, Choi SJ, Kim KW, Yu J, Ahn KM, Kim HY, Seo JH, Kwon JW, Kim BJ, Kim HB, Shim JY, Kim WK, Song DJ, Lee SY, Jang GC, Kwon JY, Lee KJ, Park $H J$, Lee PR, Won HS, Hong SJ: Association between maternal characteristics and neonatal birth weight in a Korean population living in the Seoul metropolitan area, Korea: a birth cohort study (COCOA). J Korean Med Sci 2013, 28:580-585.

18. Shin $Y H$, Yu J, Kim KW, Ahn K, Hong SA, Lee E, Yang SI, Jung YH, Kim HY, Seo JH, Kwon JW, Kim BJ, Kim HB, Shim JY, Kim WK, Song DJ, Lee SY, Jang GC, Suh DI, Yang HJ, Kim BS, Choi SJ, Oh SY, Kwon JY, Lee KJ, Park HJ, Lee PR, Won HS, Hong SJ: Association between cord blood 25-hydroxyvitamin $D$ concentrations and respiratory tract infections in the first 6 months of age in a Korean population: a birth cohort study (COCOA). Korean J Pediatr 2013, 56:439-445.

19. Yang HJ, Lee SY, Suh DI, Shin YH, Kim BJ, Seo JH, Chang HY, Kim KW, Ahn K, Shin YJ, Lee KS, Lee CM, Oh SY, Kim H, Leem JH, Kim HC, Kim EJ, Lee JS, Hong SJ: The Cohort for Childhood Origin of Asthma and allergic diseases (COCOA) study: design, rationale and methods. BMC Pulm Med 2014, 14:109.

20. Spielberger CD: Manual for the State-Trait Anxiety Inventory. Palo Alto: Consulting Psychologists Press; 1983.

21. Kim JT, Shin DK: A study based on the standardization of the STAI for Korea. New Med J 1978, 21:69-75.

22. Hahn DW, Lee CH, Chon KK: Korean adaptation of Spielberger's STAI (K-STAI). Korean J Health Psychol 1996, 1:1-14.

23. Brasch-Andersen C, Christiansen L, Tan Q, Haagerup A, Vestbo J, Kruse TA: Possible gene dosage effect of glutathione-S-transferases on atopic asthma: using real-time PCR for quantification of GSTM1 and GSTT1 gene copy numbers. Hum Mutat 2004, 24:208-214.

24. Koch A, Molbak K, Homoe P, Sorensen P, Hjuler T, Olesen ME, Pejl J, Pedersen $\mathrm{FK}$, Olsen OR, Melbye M: Risk factors for acute respiratory tract infections in young Greenlandic children. Am J Epidemiol 2003, 158:374-384.

25. Jones LL, Hashim A, McKeever T, Cook DG, Britton J, Leonardi-Bee J: Parental and household smoking and the increased risk of bronchitis, bronchiolitis and other lower respiratory infections in infancy: systematic review and meta-analysis. Respir Res 2011, 12:5.

26. luchi T, Akaike M, Mitsui T, Ohshima Y, Shintani Y, Azuma H, Matsumoto T: Glucocorticoid excess induces superoxide production in vascular endothelial cells and elicits vascular endothelial dysfunction. Circ Res 2003, 92:81-87.

27. Gilliland FD, Rappaport EB, Berhane K, Islam T, Dubeau L, Gauderman WJ, McConnell R: Effects of glutathione S-transferase P1, M1, and T1 on acute respiratory illness in school children. Am J Respir Crit Care Med 2002, 166:346-351.

28. Rebbeck TR: Molecular epidemiology of the human glutathione S-transferase genotypes GSTM1 and GSTT1 in cancer susceptibility. Cancer Epidemiol Biomarkers Prev 1997, 6:733-743.

29. Habdous M, Siest G, Herbeth B, Vincent-Viry M, Visvikis S: Glutathione S-transferases genetic polymorphisms and human diseases: overview of epidemiological studies. Ann Biol Clin 2004, 62:15-24.

30. Wright RJ: Moving towards making social toxins mainstream in children's environmental health. Curr Opin Pediatr 2009, 21:222-229.

31. Zafir A, Banu N: Modulation of in vivo oxidative status by exogenous corticosterone and restraint stress in rats. Stress 2009, 12:167-177.

32. O'Connor TM, O'Halloran DJ, Shanahan F: The stress response and the hypothalamic-pituitary-adrenal axis: from molecule to melancholia. QJM 2000, 93:323-333.

33. Nozik-Grayck E, Dieterle CS, Piantadosi CA, Enghild JJ, Oury TD: Secretion of extracellular superoxide dismutase in neonatal lungs. Am J Physiol Lung Cell Mol Physiol 2000, 279:L977-L984.

34. Kratschmar DV, Calabrese D, Walsh J, Lister A, Birk J, Appenzeller-Herzog C, Moulin P, Goldring CE, Odermatt A: Suppression of the Nrf2-dependent antioxidant response by glucocorticoids and 11 beta-HSD1-mediated glucocorticoid activation in hepatic cells. PLoS One 2012, 7:e36774.

35. Korytina GF, Yanbaeva DG, Babenkova LI, Etkina El, Victorova TV: Genetic polymorphisms in the cytochromes P-450 (1A1, 2E1), microsomal epoxide hydrolase and glutathione $\mathrm{S}$-transferase $\mathrm{M} 1, \mathrm{~T} 1$, and $\mathrm{P} 1$ genes, and their relationship with chronic bronchitis and relapsing pneumonia in children. J Mol Med 2005, 83:700-710.

36. Cohen S, Janicki-Deverts D, Turner RB, Casselbrant ML, Li-Korotky HS, Epel ES, Doyle WJ: Association between telomere length and experimentally induced upper respiratory viral infection in healthy adults. JAMA 2013, 309:699-705.

37. Shalev I, Entringer S, Wadhwa PD, Wolkowitz OM, Puterman E, Lin J, Epel ES: Stress and telomere biology: a lifespan perspective. Psychoneuroendocrinology 2013, 38:1835-1842.

38. Baldini M, Lohman IC, Halonen M, Erickson RP, Holt PG, Martinez FD: A Polymorphism* in the $5^{\prime}$ flanking region of the CD14 gene is associated with circulating soluble CD14 levels and with total serum immunoglobulin E. Am J Respir Cell Mol Biol 1999, 20:976-983.

39. Kabesch M, Hasemann K, Schickinger V, Tzotcheva I, Bohnert A, Carr D, Baldini M, Hackstein H, Leupold W, Weiland SK, Martinez FD, Mutius E, Bein $\mathrm{G}$ : A promoter polymorphism in the CD14 gene is associated with elevated levels of soluble CD14 but not with IgE or atopic diseases. Allergy 2004, 59:520-525.

40. Pincus-Knackstedt MK, Joachim RA, Blois SM, Douglas AJ, Orsal AS, Klapp BF, Wahn U, Hamelmann E, Arck PC: Prenatal stress enhances susceptibility of murine adult offspring toward airway inflammation. J Immunol 2006, 177:8484-8492.

41. Tuchscherer M, Kanitz E, Otten W, Tuchscherer A: Effects of prenatal stress on cellular and humoral immune responses in neonatal pigs. Vet Immunol Immunopathol 2002, 86:195-203.

42. Wright RJ: Perinatal stress and early life programming of lung structure and function. Biol Psychol 2010, 84:46-56.

43. Kajekar R: Environmental factors and developmental outcomes in the lung. Pharmacol Ther 2007, 114:129-145.

44. Bals R, Hiemstra PS: Innate immunity in the lung: how epithelial cells fight against respiratory pathogens. Eur Respir J 2004, 23:327-333.

45. Pelletier AJ, Mansbach JM, Camargo CA Jr: Direct medical costs of bronchiolitis hospitalizations in the United States. Pediatrics 2006, 118:2418-2423.

46. Stein RT, Sherrill D, Morgan WJ, Holberg CJ, Halonen M, Taussig LM, Wright AL, Martinez FD: Respiratory syncytial virus in early life and risk of wheeze and allergy by age 13 years. Lancet 1999, 354:541-545.

47. Bacharier LB, Cohen R, Schweiger T, Yin-Declue H, Christie C, Zheng J, Schechtman KB, Strunk RC, Castro M: Determinants of asthma after severe respiratory syncytial virus bronchiolitis. J Allergy Clin Immunol 2012, 130:91-100. e103.

doi:10.1186/1471-2466-14-154

Cite this article as: Lee et al:: The effect of perinatal anxiety on bronchiolitis is influenced by polymorphisms in ROS-related genes. BMC Pulmonary Medicine 2014 14:154

\section{Submit your next manuscript to BioMed Central and take full advantage of:}

- Convenient online submission

- Thorough peer review

- No space constraints or color figure charges

- Immediate publication on acceptance

- Inclusion in PubMed, CAS, Scopus and Google Scholar

- Research which is freely available for redistribution 